\title{
Assessment of Clinical laboratory using WHO AFRO-SLIPTA Quality Standards in a Referral Hospital laboratory in Ghana
}

Rufai Tanko ( $\sim$ rufaitanko@yahoo.com )

New Juaben South Municipal Health Directorate, Ghana Health Services

Enoch Aninagyei

Department of Biomedical Sciences, School of Basic and Biomedical Sciences, University of Health and Allied Sciences

Albert Gyau Baffour

New Juaben South Municipal Health Directorate, Ghana Health Services

Fred Gbadago

Laboratory Department, Suhum Government Hospital

Gideon Addae

Laboratory Department, Suhum Government Hospital

Donne Ameme

School of Public Health, Department of Epidemiology and Disease Control,University of Ghana

Samuel Oko Sackey

School of Public Health, Department of Epidemiology and Disease Control,University of Ghana

Kenu Ernest

School of Public Health, Department of Epidemiology and Disease Control,University of Ghana

\section{Research Article}

Keywords: Laboratory, Quality Management, Quality System Essentials, Assessment, Ghana

Posted Date: June 17th, 2021

DOl: https://doi.org/10.21203/rs.3.rs-322208/v1

License: (c) (i) This work is licensed under a Creative Commons Attribution 4.0 International License.

Read Full License 


\section{Abstract}

Introduction: The hospital laboratory exists to provide accurate and reliable results to physicians for effective management of patients. The Quality Management System (QMS) in the laboratory must be effective and efficient so that reliable results will be produced for patient care. Quality System Essentials together with International Organization for Standardization (IOS) 15189 are the core components used to establish a QMS in the Laboratory. Laboratory assessments are an effective means to determine whether a laboratory is providing accurate and reliable results and adhering to good laboratory practices. We assessed the Suhum Hospital laboratory to identify gaps in the components of Quality System Essentials.

Method: This exploratory study investigated the practice of QMS in Suhum Hospital laboratory, a public district hospital laboratory in Ghana, in January 2016. Data collected comprised activities by laboratory staff during onsite visits and evidence of documentation. Permission to collect data was sought from the Hospital Management and Laboratory Manager. We used the WHO-AFRO laboratory Strengthening Checklist to assess the laboratory. We reviewed the records to verify if the laboratory quality manuals, policies, logs, Standard Operating Procedures (SOPs) were complete, current, accurate, and annually reviewed. Finally, we interviewed staff of the laboratory and clinicians to obtain information to learn their perspective on the laboratory's performance.

Results: The undepartmentalized laboratory is headed by a manager with a staff strength of 13 trained professionals. It receives averagely 100 patient samples daily and runs 30 different tests. The main strengths of the laboratory in terms of Quality System Essentials were information management 15/18 (83\%), process control $25 / 33(77 \%)$, internal audit $6 / 10(60 \%)$, facilities and safety $25 / 43(58 \%)$, purchasing and inventory $17 / 30$ (57\%). However, equipment management $8 / 30(27 \%)$, occurrence management $3 / 12(25 \%)$, documents and records $7 / 25(28 \%)$, organization and personnel $8 / 20(40 \%)$, corrective actions 5/12 (42\%) and client management 5/17 (29\%) required improvement.

Conclusion: Findings from the laboratory audit pointed to an overall weak laboratory QMS based on WHO/AFRO laboratory strengthening checklist and rating. Concerted efforts are therefore required to improve the laboratory QMS rating to improve quality of care to clients in all district hospital laboratories in Ghana.

\section{Introduction}

Clinical laboratory testing plays a crucial role in the detection, diagnosis, and treatment of disease in patients [1]. Almost $60-70 \%$ treatment and management decisions depend on laboratory findings [2]. Unfortunately, the use of laboratory evidence-based patient healthcare remains a complex problem in many countries, especially in sub-Saharan Africa [3]. Laboratories in developing settings are, however, often under-resourced resulting in poor performance. Usually, laboratory results are perceived by some clinicians to be unreliable, especially when they are found to be discordant with clinical judgement [4]. 
Sub-standard quality assurance or quality control procedures in a laboratory can lead to errors in testing and subsequent inaccurate results. These can have an adverse and potentially lethal impact on patients as well as laboratory-based public health surveillance and monitoring of reportable diseases [5].

However, a well-defined quality system is a must for ensuring the quality of services [6]. It ensures the accuracy of test results, increases the confidence of patients, clinicians, and communities in the value of laboratory testing [7]. The Quality System Essentials (QSE) together with International Organization for Standardization (ISO) 15189, are the core components used to establish a Quality Management System (QSM) in the laboratory. It covers the entire system of laboratory testing including pre-analytical, analytical, and post-analytical phases. These components are organization, personnel, documents and records, equipment's, purchasing and inventory management, review, process control/ external and internal quality control, information management, occurrence management, customer service and satisfaction, internal audit, facilities and safety. Conformity to these were assessed based on scores of zero to a five-star Stepwise Laboratory Improvement Process Towards Accreditation (SLIPTA) grade, where 0 Star (0-142 points; <55\%), 1 Star (143-165 points; <55-64\%), 2 Stars (166-191 points; 65-74\%), 3 Stars (192-217 points; 75-84\%), 4 Stars (218-243 points; 85-94\%) and 5 Stars (244-258; $\geq 95 \%$ ) [8]. Although laboratory accreditation guarantees the ability to perform with high quality standards; accredited laboratories in Africa remain scarce [9].

World Health Organization Regional Office for Africa (WHO AFRO) has established the SLIPTA to strengthen the laboratory systems of its member states [10]. It is a process that enables laboratories to develop and document their ability to detect, identify, and promptly report all diseases of public health significance that may be present in clinical specimens [10].

Laboratory assessments are an effective means to determine whether a laboratory is providing accurate and reliable results and adhering to good laboratory practices. We assessed the baseline laboratory quality standards in Suhum Government Hospital to identify gaps in the components of QSE and make recommendations to strengthen its operations.

\section{Method}

\subsection{Study Area}

The study was carried out in Suhum Municipality of the Eastern Region of Ghana. The Suhum Municipality population is about 105,605 projected from the 2010 Population and Housing Census (2010 Population and Housing Census Report). It covers an area of $450 \mathrm{~km}^{2}$ and is located in the southern part of the Eastern Region. Suhum is about 60 kilometers North - West of Accra. The municipality shares boundaries with East Akim to the North, Ayensuano to West and South, Akwapim North municipality to the East and New Juaben Municipality to North East (Figure 1).

There are three (3) private health facilities, three (3) prayer and healing centres, three (3) major pharmacies, forty-four (44) chemical sellers, and thirty-two (32) CHPS zones in the municipality. The 
Suhum Government Hospital is the main referral center for all privates and health centres in the municipality; therefore, the laboratory serves as the referral laboratory for all hospitals, clinics, health centers, etc. in the municipality

Figure 1: Map of Suhum Municipality and Sub-municipal [11]

\section{Study design}

This exploratory study investigated the practice of QMS in Suhum Hospital laboratory, a public district hospital laboratory in Ghana, in January 2016. Data collected comprised activities by laboratory staff during onsite visits and evidence of documentation (policies, processes, and procedures).

\section{Preparation for the Assessment}

The facility was given notification prior to the audit through the Medical Superintendent, the Administrator, and the Laboratory Management. The checklist for the assessment (WHO AFRO SLIPTA) was given to the facility to study and prepare for the audit. Quality Manuals were requested from the laboratory on the day of assessment. We used the WHO-AFRO laboratory Strengthening Checklist to assess the laboratory. We reviewed the records to verify if the laboratory quality manuals, policies, logs, Standard Operating Procedures (SOPs), and other manuals were complete, current, accurate, and regularly reviewed. We also observed laboratory operations to ensure practice matched with the written procedures in all phases of laboratory testing and the processes were appropriate for the testing performed. We interviewed staff of the laboratory to obtain information and interviewed clinicians to learn their perspective on the laboratory's performance.

\section{Access to the Facility}

Introductory meeting with the laboratory staff was held to introduce the audit plan on the day of assessment. The nature and expectations of the audit team were discussed.

\section{Presentation of Findings}

A brief final closing meeting was held after the preliminary audit report to present and discuss findings. Major and minor non-conformities identified were discussed at the meeting. Laboratory staff were given the opportunity to respond to the findings. After accepting the findings, a final report was submitted to the laboratory and hospital management. The laboratory staff agreed to address the nonconformity and management pledged their support to fill the gaps.

\section{Quality management systems in the laboratories}

A checklist developed by the World Health Organization in the African Region for Stepwise Laboratory Quality Improvement Process Towards Accreditation (WHO AFRO SLIPTA) was used to collect data from the field. Data was collected through observations and interviews. The checklist contained 12 sections with 110 questions worth 258 points. These sections are categorized as: "Documents and Records", 
"Management Reviews", "Organization and Personnel", "Client Management and customer service”, "Equipment", "Internal Audit", "Purchasing and Inventory", "Process Control and Quality Assessment", "Information Management", "Corrective Action”, "Occurrence/Incident Management and Process Improvement", "Facilities and Safety". Each laboratory was graded from zero to five stars based on the total audit score. Total audit score of < 55\% (0-142 points) represents Zero Star; $143-165$ points (55-64\%) represents 1 Star; $166-191$ points (65-74\%) represents 2 Stars; $192-217$ points (75-84\%) represents 3 Stars; $218-243$ points (85-94\%) represents 4 Stars and $244-258$ points $(\geq 95 \%)$ represents 5 Stars. The scoring system in the checklist has three sections designated as; $Y=Y e s, P=P a r t i a l, N=$ No. Each item (question) has options $\mathrm{Y}, \mathrm{P}$, or $\mathrm{N}$. Each item has been awarded a point value of 2, 3, 4, or 5 points based upon relative importance and/or complexity. Some items have certain elements (sub-questions). All elements of an item must be satisfactorily presented to indicate "Yes" and thus award the corresponding points. Items marked " $\mathrm{P}$ " and " $\mathrm{N}$ " receive 1 point and 0 point, respectively [6].

\section{Gaps in quality system essentials (QSEs) in laboratory practice}

The twelve sections of the SLIPTA checklist encompassed the QSEs. Gaps were identified based on the audit report of the checklist. Gaps were non-conforming policies, processes, and procedures compared to the standard (WHO AFRO SLIPTA).

\section{Ethical considerations}

Approval for this study was granted by the Ghana Field Epidemiology and Laboratory Training

Programme (GFELTP); run by both the Ghana Health Service and the School of Public Health -

University of Ghana. Permission was sought from Hospital Management and Laboratory Managers offered their consent before the data was collected.

\section{Data Analysis Plan}

The scores were summarized and results presented as relative frequencies using Epi info version7.

\section{Results}

The laboratory has not been divided in to units or departments (undepartmentalized). The tests that were conducted include; hematology, clinical biochemistry, microbiology and blood banking. The laboratory lacked reception where all specimens were to be received. The laboratory receives an average of 100 patient samples daily and runs about 30 different tests every day.

The main strengths of the laboratory in terms of Quality System Essentials were information management 15/18 (83\%), process control/internal/external quality assessment 25/33 (77\%), internal audit $6 / 10$ (60\%), facilities and safety $25 / 43$ (58\%), purchasing and inventory $17 / 30(57 \%)$. However, equipment management $8 / 30(27 \%)$, occurrence management $3 / 12(25 \%)$, documents and records $7 / 25$ 
(28\%), organization and personnel $8 / 20(40 \%)$, corrective actions 5/12 (42\%) and client management $5 / 17(29 \%)$ required improvement (Table 1$)$.

\section{Organization and personnel}

The laboratory has thirteen cadres of staffs made up of medical laboratory scientists, laboratory technical officers, laboratory assistance, casuals, interns, and orderlies. The laboratory has no organizational chart and therefore there were no documented lines of authority and reporting system, but there was a duty roster. The laboratory is being headed by a Deputy Medical Laboratory Scientist and there is a Quality Control Manager who monitors the quality system of the laboratory but has not been adequately trained. There was no system for competency assessment and staffs did not have adequate training policies, procedures, or training plans. There were no personnel files at the department but present at the hospital's general office and no documentation on the orientation of staffs.

Daily routine tasks were partially assigned. Personnel do not document adverse events like incidence of nonconformance with established laboratory protocols which may result in patient harm or misdiagnosis. Staff meetings were not held regularly to ensure communication within the laboratory and no recorded notes to facilitate review of progress.

\section{Documents and records}

At the makeshift sample collection point, there was a register in which all specimens received were recorded and given a unique identification number. There was also a log book in which test results together with patient information were recorded. Few bench aids, which have not been reviewed for many years, were available and well displayed for easy reference. There was no proper inventory control system as well as no mechanism for resolution of complaints and feedback. Additionally, the laboratory lacks essential documents such as quality and safety manuals and formats for corrective and preventive actions. There were SOPs available for all procedures except in the hematology section of the laboratory. Finally, the archived results were stock piled in a cupboard and were not locked to ensure confidentiality.

\section{Equipment}

The laboratory has some of the basic equipment required. The departments have some automated and semi- automated analyzers to facilitate the testing procedures. Temperature charts were available for equipment that requires temperature control. However, equipment inventory data was not available for most equipment in the laboratory and there was also no equipment maintenance record. There was, however, no documented program of preventive maintenance that follows the manufacturer's recommendations. Routine calibration of laboratory equipment was not scheduled and indicated on the equipment. Non- functioning equipment's were not labelled and were still found in the laboratory. There was no contingency plan such as back- up procedures for equipment failure. Manufacturer's operator manual was not available for some of the equipment. There was frequent interruption of testing services in the department due to intermittent power supply, reagents out of stock and equipment breakdown. 


\section{Purchasing and Inventory}

There was a procurement officer in the central administration of the hospital who controls all issues of procurement in the hospital and so the laboratory has no dedicated procurement officer. At the laboratory unit, there was an inventory control system, but the records were incomplete. Reviews of the system were done annually and contact information of suppliers was available at the central procurement unit. However, testing services in the department were sometimes interrupted due to lack of reagents and broken-down equipment. The First-Expiry-First-Out system for reagent usage was partially practiced. Expired products were not labelled and disposed properly.

\section{Management Review}

There was no work plan for the laboratory, however, the budget for the laboratory was run by the central administration and requests are forwarded through hierarchy. Quality and technical records were reviewed by the head of the department; however, this was partially done. Information on malaria, sickling, and sputum were the only records available for internal quality control. There were no records on the annual reviewing of quality systems at management review meetings.

\section{Process control and Internal and External quality assessment}

There were internal quality controls for some procedures in the laboratory which were performed and documented, and some equipment were run with internal controls. These quality control results were usually not monitored and reviewed for shift or trend analysis. There were no temperature charts and thermometers for monitoring temperature-dependent equipment. Primary samples lacking proper identification were processed, and when rejected no records were kept. Sample aliquots were not kept and all samples were discarded after the results have been released. The laboratory had communicated transportation requirements to sample submitters, but this was done verbally and had no program in place for monitoring transportation samples. There were few procedure manuals in the working area, but the reagent log book was not available for all reagents received in the laboratory. Test results were sometimes validated and interpreted by authorized personnel. The laboratory, however, took part in External Quality Assessment programs for TB microscopy and CD4 counts and the results from the external assessors were commendable.

\section{Information management}

Test results were verified by authorized personnel and confirmed with patient identity. The technical staff were not identified on the requisition and result forms, the only form of identification was their signature on the report form. There was no system for review of clerical errors on the test results. All test results were recorded in log books with no back-ups for the stored data. Moreover, the archived results were not stored in a secure location and there was no policy and procedure for the protection of confidential information. There was also no codification of the patient's samples and results to ensure confidentiality. 
There was no occurrence/incidence book where incidences were recorded when they occurred. There was also no policy in place for root cause analysis and no corrective action or preventive action records were kept to prevent reoccurrence. There was no system in place to evaluate client satisfaction and patient results were not confidential, as the laboratory staff knew the full names of the patients.

\section{Internal audit}

Internal audit was carried out by laboratory and non-laboratory personnel but it was not documented. Recommendations for corrective action based on the findings were not available.

\section{Facility and Safety}

The laboratory working space was not adequate and not separated from one another for optimal workflow. Work areas were cleaned and daily disinfection was done. Personal protective equipment was easily accessible at work stations and were utilized appropriately and consistently. Sharps were handled properly and disposed in sharp containers. Post-exposure prophylaxis policies and procedures were accessible in the laboratory. Some staff had been vaccinated against Hepatitis B virus infection. There were no biosafety cabinets to process infectious materials. The major challenges for safety identified include an appointed biosafety officer who had not been trained to implement a safety program in the laboratory. There was no safety signage in some work areas and entry into the laboratory was not restricted. Waste bins were not labeled and they were without the appropriate rubber lining for waste segregation. There was poor storage of hazardous chemicals. Moreover, safety inspections or audits were not conducted regularly and documented.

\section{Table 1. Quality system essentials scores for Suhum Hospital Laboratory}




\begin{tabular}{|c|c|c|}
\hline Sections & Total score & Laboratory score (\%Score) \\
\hline Documents \& Records & 25 & $7(28)$ \\
\hline Management Reviews & 17 & $5(29)$ \\
\hline Organization \& Personnel & 20 & $8(40)$ \\
\hline Client Management \& Customer Service & 8 & $3(38)$ \\
\hline Equipment & 30 & $8(27)$ \\
\hline Internal Audit & 10 & $6(60)$ \\
\hline Purchasing \& Inventory & 30 & $17(57)$ \\
\hline Process control \& Quality Assessment & 33 & $25(77)$ \\
\hline Information Management & 18 & $15(83)$ \\
\hline Corrective Action & 12 & $5(42)$ \\
\hline Occurrence/Incidence Management & 12 & $3(25)$ \\
\hline Facilities \& Safety & 43 & $25(58)$ \\
\hline Total & 258 & $127(49)$ \\
\hline
\end{tabular}

Key:

1. Pts $=$ Points scored $(n)$.

2. Actual Score $=N$

3. \%Score (Percentage Score) $=\mathrm{n} / \mathrm{N}$

\section{Discussion}

The role of a clinical laboratory is to promptly provide highly reliable laboratory data to satisfy the needs of clinicians involved in medical practice and health maintenance of patients[12]. It is therefore vital to minimize the chance for errors and to ensure the highest possible quality of testing and results. It is expected that laboratory managers, supervisors, and testing officers should be well versed and knowledgeable about all aspects of the quality management systems in their laboratory operations. In assessing the components of the QSEs, the laboratory was found to be doing well in information management, process control/internal/external quality assessment, and internal audit. It was done averagely in facilities and safety, purchasing, and inventory but needed improvements in equipment management, occurrence management, documents and records, organization and personnel, corrective actions, and client management. 
Ghana adopted SLIPTA a decade ago, but the implementation started with only national and regional laboratories. All district and subdistrict laboratories were excluded from the programme. In Eastern region, only the Regional Hospital Laboratory was involved in the programme and was graded "4 star" laboratory through an audit by ASLM in 2014 [13].

The SLIPTA score for the current study facility was $49 \%$ which was $<55 \%$ ( $0-142$ points). This score conforms to previous studies in Ghana [13,14], and in Ethiopia [15] at baseline. Nonetheless, compared to previous reports in the country, none of the facilities enrolled in the SLIPTA programmes audited by ASLM scored $<55 \%$ (0-142points). In Ghana, the low SLIPTA score recorded could be attributed to underresourced and lack of involvement of the district laboratories in QMS. Other contributing factors are lack of management commitment in the laboratories, lack of planning, implementation, and monitoring of laboratory policies, processes, and procedures. It is obvious that district laboratories must also be strengthened to provide accurate, trusted, and reliable results for good healthcare delivery. Meanwhile, improvement and maintenance of the quality of the laboratory staff and environment are essential to achieve this goal[12]. Another challenge faced by many clinical laboratories is staff motivation, especially given the paucity of opportunities for advancement in the laboratory setting[16].

Prior to the eventual declaration of the Covid-19 outbreak pandemic, WHO encouraged countries to intensify testing. The only way nations can adhere to the advice is to depend on Medical Laboratory systems already available in their respective countries. Unfortunately for Ghana, the laboratories were poorly resource due to the neglect of the sector by the authorities over the years. There is no gainsaying to the fact that medical laboratories are the least to be considered in the design of a health care facility and healthcare authorities hardly see any importance in the involvement of the practitioners of medical laboratory science in topical issues regarding the subsector.

Hence, key stakeholders' engagement such as Ministry of Health, Ghana Health Service, Allied Health Professional Council and Hospital management commitment are critical in laboratory service delivery. It is prudent that all laboratories are mentored and trained to establish QMS and to achieve quality improvement $[17,18]$. These will create awareness and understanding to implement quality systems in clinical laboratories. The baseline information provided by this study will therefore help devise strategies for QMS implementation in various district health facilities in the region. It is a known fact that delay in correct diagnosis, treatment complications, exposure of patients to side effects of antibiotics, unnecessary cost, upsurge of drug resistance, and failure to provide proper treatment are some of the devastating consequences.

\section{Conclusion}

The Laboratory Quality Management System in the study health facility was found to be weak (per total points scored), the facility was zero star rated, based on WHO/AFRO laboratory strengthening checklist and rating. This implies that laboratory results from this facility may not be reproducible or accurate. The study strongly recommends that the Government of Ghana, Ministry of Health, Ghana Health Service, and 
other responsible agencies focus on the strengthening and involvement of all district laboratories in the region to gradually implement QMS and for quality healthcare delivery.

\section{Declarations}

\section{Data Availability}

All data have been fused into the manuscript and it's supporting information files.

\section{Conflict of Interest}

The authors declare that they have no conflicts of interest

\section{Acknowledgement}

The authors wish to acknowledge the Ghana Field Epidemiology Training Program (GFELTP) for their contributions towards the success of this laboratory assessment and to the laboratory staff and management of Suhum Government Hospital.

\section{Funding}

This work was funded from the authors' own resources with support from our collaborators acknowledged above.

\section{Authors' contributions}

The authors' contributions were as follows: conceptualization, RT, EK, DA, SOS.; methodology, RT, EA, AGB, FG, GA; formal analysis, RT; writing-original draft preparation, RT; writing-review and editing, RT, EK, DA, and SOS; supervision of study, EK, SOS and DA. All authors have read and approved the manuscript.

\section{References}

[1] T. F. Peter, P. D. Rotz, D. H. Blair, A.-A. Khine, R. R. Freeman, and M. M. Murtagh, "Impact of Laboratory Accreditation on Patient Care and the Health System," Am. J. Clin. Pathol., vol. 134, no. 4, pp. 550-555, Oct. 2010, doi: 10.1309/AJCPH1SKQ1HNWGHF.

[2] M. Regan and R. Forsman, "The impact of the laboratory on disease management," Dis. Manag., vol. 9, no. 2, pp. 122-130, 2006.

[3] L. W. Niessen, E. W. Grijseels, and F. F. Rutten, "The evidence-based approach in health policy and health care delivery," Soc. Sci. Med., vol. 51, no. 6, pp. 859-869, 2000. 
[4] L. R. Andiric and C. G. Massambu, "Laboratory Quality Improvement in Tanzania," Am. J. Clin. Pathol., vol. 143, no. 4, pp. 566-572, Apr. 2015, doi: 10.1309/AJCPAB4A6WWPYIEN.

[5] S. R. Blumen, S. Naud, M. V. Palumbo, B. Mclntosh, and B. W. Wilcke, "Measuring the Application of Quality System Essentials in Vermont Clinical Laboratories," Lab. Med., vol. 42, no. 3, pp. 151-157, Mar. 2011, doi: 10.1309/LML7NIALOXGPCIFJ.

[6] D. P. Silva, "Guidelines on Establishment of Accreditation of Health Laboratories," p. 49, 2007.

[7] W. H. Organization, Global tuberculosis report 2013. World Health Organization, 2013.

[8] C. Shaw, O. Groene, N. Mora, and R. Sunol, "Accreditation and ISO certification: do they explain differences in quality management in European hospitals?," Int. J. Qual. Health Care, vol. 22, no. 6, pp. 445-451, 2010.

[9] I. M. Taremwa et al., "Assessment of three medical and research laboratories using WHO AFRO_SLIPTA Quality Standards in Southwestern Uganda: long way to go," Pan Afr. Med. J., vol. 28, 2017, doi: 10.11604/pamj.2017.28.129.10995.

[10] J.-B. Ndihokubwayo et al., "Implementation of the World Health Organization Regional Office for Africa stepwise laboratory quality improvement process towards accreditation," Afr. J. Lab. Med., vol. 5, no. 1, pp. 1-8, 2016.

[11] B. T. Boakye, "Assessing the Quality of Expanded Programme on Immunization (EPI) Data: A Case Study in the Suhum Muncipality of the Eastern Region," PhD Thesis, University of Ghana, 2019.

[12] H. Yokota and Y. Yatomi, "Future roles of clinical laboratories and clinical laboratory technologists in university hospitals," Rinsho Byori, vol. 61, no. 8, pp. 686-691, 2013.

[13] E. Agboli et al., "Gaps in Laboratory Quality Management Systems in the Volta Region of Ghana," Asian J. Med. Health, vol. 10, no. 1, pp. 1-9, Feb. 2018, doi: 10.9734/AJMAH/2018/39254.

[14] B. Nkrumah et al., "Building local human resources to implement SLMTA with limited donor funding: The Ghana experience," Afr. J. Lab. Med., vol. 3, no. 2, 2014.

[15] T. M. Hiwotu et al., "Laboratory system strengthening and quality improvement in Ethiopia," Afr. J. Lab. Med., vol. 3, no. 2, 2014.

[16] L. Samuel and S. Novak-Weekley, "Commentary: The Role of the Clinical Laboratory in the Future of Health Care: Lean Microbiology," J. Clin. Microbiol., vol. 52, no. 6, pp. 1812-1817, 2014.

[17] S. M. Tamil and A. Srinivas, "Evaluation of quality management systems implementation in medical diagnostic laboratories benchmarked for accreditation," J. Med. Lab. Diagn., vol. 6, no. 5, pp. 2735, Aug. 2015, doi: 10.5897/JMLD2015.0104. 
[18] T. Maruta, P. Rotz, and T. Peter, "Setting up a structured laboratory mentoring programme," Afr. J. Lab. Med., vol. 2, no. 1, p. 7 pages, Mar. 2013, doi: 10.4102/ajlm.v2i1.77.

Figures

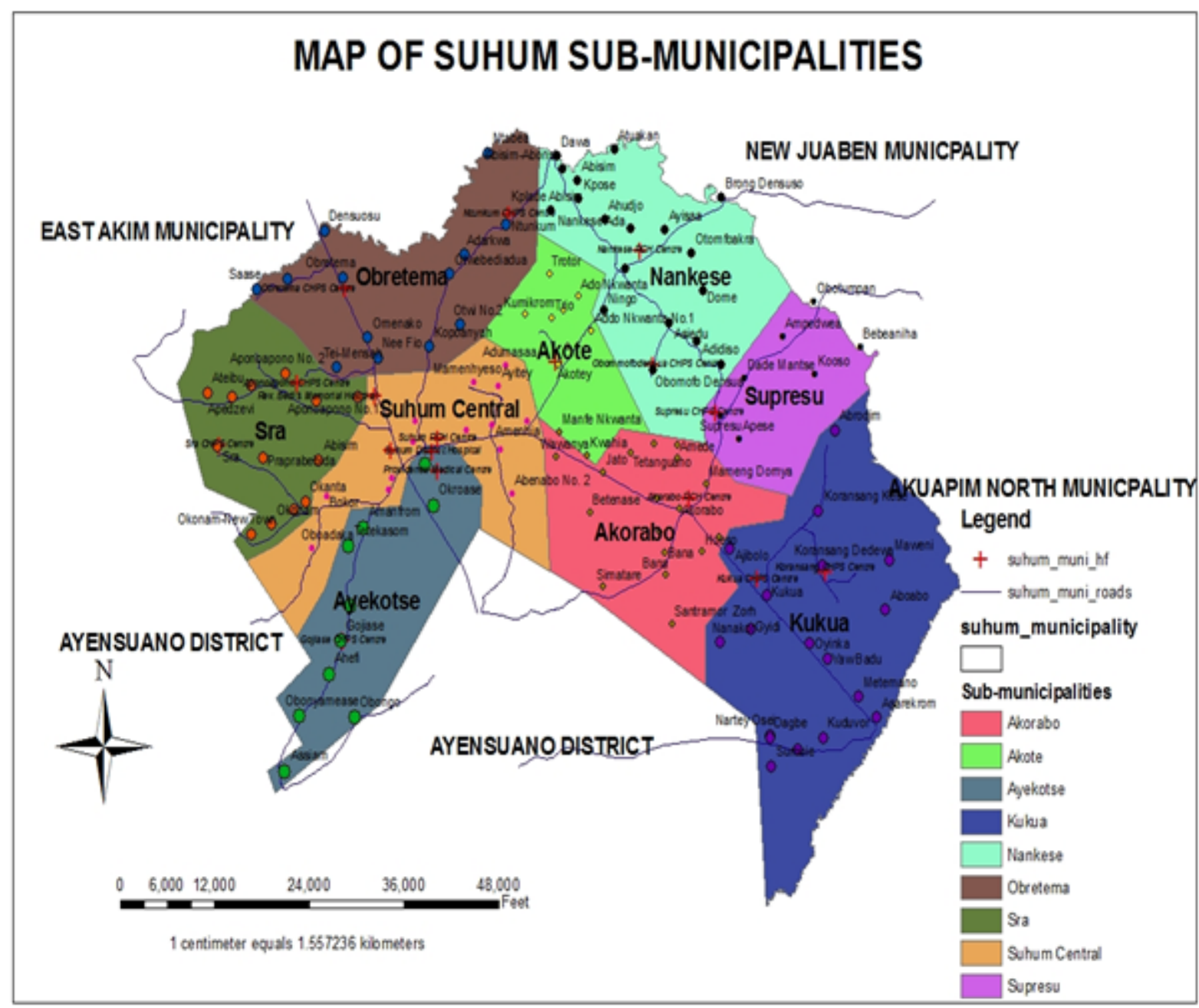

Figure 1

Map of Suhum Municipality and Sub-municipal [11] 